\title{
An Octave Bandwidth Metamaterials-based Hexagonal Patch Antenna
}

\author{
Dong Sik Woo ${ }^{1}$, Cherl-Hee Lee ${ }^{1}$, Kang Wook Kim ${ }^{1}$, Hyun-Chul Choi ${ }^{1}$ \\ ${ }^{I}$ School of Electronics Engineering, Kyungpook National University, \\ 80 Daehak-ro, Buk-gu, Daegu, 702-701, Korea \\ hcchoi@ee.knu.ac.kr
}

\begin{abstract}
A broadband metamaterials (MTM)-based hexagonal patch antenna is designed and presented. A hexagonal shape of a top patch on a mushroom structure makes not only direct-current paths between two ends of the patch but also round-current path along the outside of the patch, thereby widening a resonance frequency of the mushroom MTM antenna. Moreover, input microstrip transmission line acted as a reflecting element for the surface wave, resulting in forward-directed end-fired radiation. The hexagonal patch was implemented by utilizing a composite right-and left-handed (CRLH) transmission line. The antenna operates from $15.9 \mathrm{GHz}$ to $30.6 \mathrm{GHz}$ covering the $\mathrm{Ku}$ - to $\mathrm{Ka}$-band. The antenna gain is from $6 \mathrm{dBi}$ to $9.3 \mathrm{dBi}$ with small size compared to the conventional antennas. The application areas are in automotive radars and ground penetrating radar (GPR).
\end{abstract}

Index Terms-Metamaterials antenna, broadband antennas, microstrip antennas, ground penetrating radar (GPR).

\section{INTRODUCTION}

The Ground penetrating radar (GPR) is a well-known technology and has been found to be a special option for wide range applications, including archaeology, geophysical research, mine or buried object detection and so on [1].

Ultra-wideband antennas are one of the most critical parts of broadband GPR system. Most printed and planar antennas have gained interest due to their small size, low cost and low weight, ease of fabrication. But certain types have some disadvantage such as narrow frequency bandwidth and poor in robustness. Dispersive or non-dispersive antennas have been commonly used for GPR systems, such as dipole antenna Bow-tie antenna, TEM horn antenna, Vivaldi or tapered slot antenna (TSA), and equiangular spiral antenna [2]. However, these are not compatible with high frequency microwave integrated circuits (MICs) because of their non-planar structure and electrically large size. Moreover, the input impedance of the conventional balanced antennas is more than $100 \Omega$, which is difficult in broadband impedance matching with $50 \Omega$ feed line because of the frequency limitation of the transition or balun [3].

Recently, metamaterials (MTM) which simultaneously

Manuscript received April 29, 2015; accepted August 11, 2015.

This research was supported by the MSIP (Ministry of Science, ICT \& Future Planning), Korea, under the C-ITRC (Convergence Information Technology Research Center) support program (NIPA-2014-H0401-141004) supervised by the NIPA (National IT Industry Promotion Agency.) This study was supported by the BK21 Plus project funded by the Ministry of Education, Korea (21A20131600011). have negative permittivity $(\varepsilon)$ and permeability $(\mu)$ theoretically speculated in 1960s by the Russian physicist Viktor Veselago have received great attention due to negative refraction, reverse Cerenkov radiation, and slow light [4]-[6]. Due to these unique properties, MTM have been widely applied as microstrip patch antennas in military and commercial system applications [7]. MTM microstrip patch antennas have many attractive features such as anti-parallel phase velocity to group velocity and infinite wavelength at a certain frequency, as well as small size, low profile and integration with planar surfaces. However, they inherently have narrow frequency bandwidth, typically $1 \%-5 \%$, limiting its many attractive features. Currently, mushroom structures have been demonstrated as an effective way to increase the bandwidth of MTM microstrip patch antennas with the techniques of the stacked top patches, inserting slits or slots in addition to increasing patch height and decreasing substrate permittivity [8], [9]. The stacked patches and the conventional techniques have solved the bandwidth problem with a relatively large antenna thickness. However, there is a practical limit on increasing the antenna thickness. Therefore, bandwidth extension technique of MTM antenna without increasing the volume or degrading the performance of low-profile is a critical approach.

In this paper, we present a mushroom-like MTM hexagonal patch antenna to improve the bandwidth and directivity. Conventional mushroom-like MTM antennas have narrow bandwidth. However, a hexagonal shape of a top patch on a mushroom structure makes not only direct-current paths between two ends of the patch but also round-current path along the outside of the patch, and hence it can widen a resonance frequency of the mushroom MTM antenna. Moreover, by using of open CRLH TL structure, a radiated leaky-wave $(\mathrm{LW})$ mode is achieved where $\beta=+k_{0}[10]$. Therefore, broadside-to-endfire scanning capability was also provided. The proposed antenna was modeled by utilizing a composite right-and left-handed (CRLH) transmission line, and provided $6 \mathrm{dBi}$ to $9.3 \mathrm{dBi}$ of the antenna gain and a reduced small size compared to a conventional microstrip patch antenna. An octave bandwidth was achieved with VSWR less than 2:1.

\section{ANTENNA DESIGN}

The concept of composite right/left-hand (CRLH) MTM is introduced by Caloz et al. [11]. MTM are effective 
homogeneous structures that can be modeled by one-dimensional transmission lines (TLs) of CRLH structures under the condition that the average cell size be smaller than the guided wavelength. A general CRLH TL unit-cell consists of a series capacitance and a shunt inductance, as well as a series inductance and a shunt capacitance.

The CRLH TL model can be represented as a combination of a per-unit-length series inductance $\left(\mathrm{L}_{\mathrm{R}}{ }^{\prime}\right)$, a per-unit-length shunt capacitance $\left(\mathrm{C}_{\mathrm{R}}{ }^{\prime}\right)$, a times-unit-length shunt inductance $\left(\mathrm{L}_{\mathrm{L}}{ }^{\prime}\right)$, and a per-unit-times length series capacitance $\left(\mathrm{C}_{\mathrm{L}}{ }^{\prime}\right)$, as shown in Fig. 1. According to lossless transmission line theory, the propagation constant of a CRLH TL is given by $\gamma=j \beta=\sqrt{Z^{\prime} Y^{\prime}}$, where $Z^{\prime}$ and $Y^{\prime}$ are the per-unit-length impedance and per-unit-length admittance, respectively. $Z^{\prime}$ and $\mathrm{Y}^{\prime}$ are obtained as [11]:

$$
\begin{aligned}
& Y^{\prime}(\omega)=\frac{Y(\omega)}{\Delta z}=j\left(\omega C_{R}^{\prime}-\frac{1}{\omega L_{L}^{\prime}}\right), \\
& Z^{\prime}(\omega)=\frac{Z(\omega)}{\Delta z}=j\left(\omega L_{R}^{\prime}-\frac{1}{\omega C_{L}^{\prime}}\right) .
\end{aligned}
$$

The series resonance frequency and shunt resonance frequency are given as $\omega_{\mathrm{se}}=1 / \sqrt{\mathrm{L}_{R}^{\prime} \mathrm{C}_{L}^{\prime}}$ and $\omega_{\mathrm{sh}}=1 / \sqrt{\mathrm{L}_{R}^{\prime} \mathrm{C}_{L}^{\prime}}$, respectively.

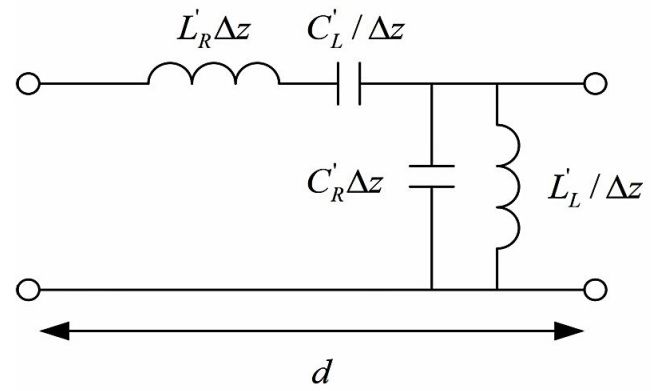

Fig. 1. Equivalent circuit model of composite right/left-handed (CRLH) MTMs TL.

For an open-ended CRLH TL, the resonant condition of $\beta_{m} L=m \pi$ must be satisfied, where $m=0, \pm 1, \pm 2, \pm 3, \ldots$ $\pm(N-1)$ is the resonance mode. For a $N$-cell CRLH TL, the total length of the CRLH TL, $L=N \times d$, can be obtained, where $d$ is the length of the unit cell; therefore,

$\frac{m \pi}{N}=\cos ^{-1}\left\{1-\frac{1}{2}\left\{\frac{\omega^{2}}{\omega_{R H}^{2}}+\frac{\omega_{L H}^{2}}{\omega^{2}}-\left(\frac{1}{\omega_{s h}^{2}}+\frac{1}{\omega_{s e}^{2}}\right) \omega_{L H}^{2}\right\}\right\}$,

where $\omega_{\mathrm{RH}}=1 / \sqrt{\mathrm{L}_{\mathrm{R}}^{\prime} \mathrm{C}_{\mathrm{R}}^{\prime}}$ and $\omega_{\mathrm{LH}}=1 / \sqrt{\mathrm{L}_{\mathrm{L}}^{\prime} \mathrm{C}_{\mathrm{L}}^{\prime}}$.

As the resonance mode number $(m)$ is zero, the CRLH TL supports zero propagation constant. Therefore, an infinite wavelength is obtained, and the length of the CRLH TL becomes independent of the resonance condition. In this case of the open-ended CRLH TL, the zeroth-mode frequency is determined by the inductance and the capacitance loaded in the CRLH TL. When a zeroth-order resonator is used for the antenna, it inherits independence of physical size, leading to one degree of freedom in designing an antenna smaller than a conventional patch antenna with a $\lambda / 2$ field distribution.

On the other hand, any open CRLH TL structure can operate as a radiated leaky-wave (LW) mode since the CRLH dispersion curve always going into radiation region. From the $\omega-\beta$ diagram, endfire radiated $\mathrm{RH}$ leaky mode is achieved where $\beta=+k_{0}$. Therefore, broadside-to-endfire capability can be provided by an open CRLH structure. Another advantage of the CRLH LW antenna feeding method is simple and broadband.
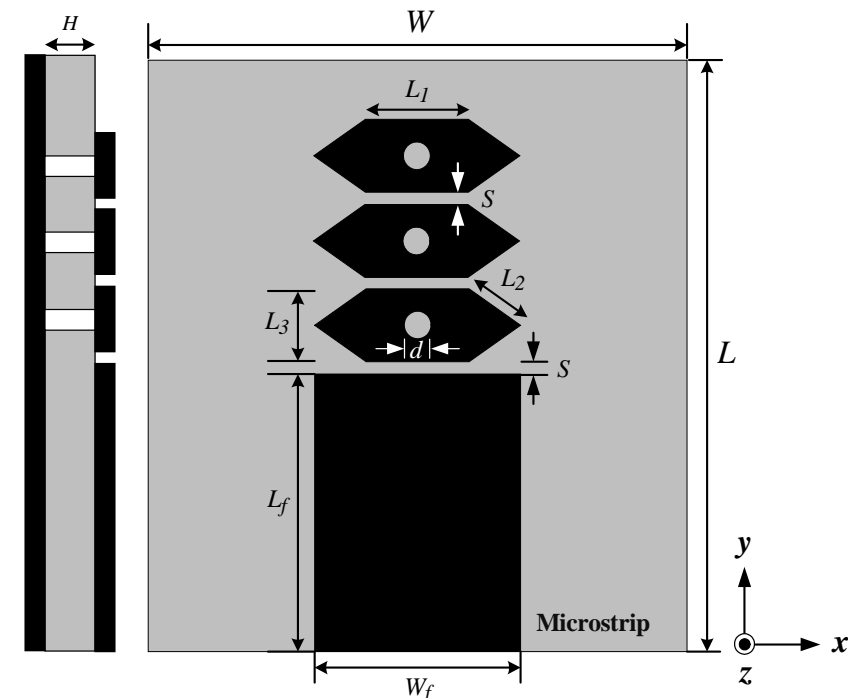

Fig. 2. Antenna geometry: $L_{\mathrm{f}}=10, W_{\mathrm{f}}=7.65, L_{1}=3.8, L_{2}=2.4, L_{3}=2.6, S=$ $0.44, d=1, H=2.37, W=20$ and $L=21$ (all dimensions are in millimeters).

Figure 2 shows the presented microstrip MTM mushroomlike antenna geometry with a hexagonal-shaped top patch. The mushroom structure is built with a hexagonal top patch and a metal via connecting the top patch to the ground. The gap between neighboring patches represents the left-handed series capacitance, and the vertical via of the top plate acts as the left-handed shunt inductance in the equivalent circuit model, while the shunt capacitance and series inductance, representing the right-handed TL, are due to parasitic effects caused by the microstrip geometry. The electrical length $L_{3} / 2$ of the RH TL is $\pi / 4 \mathrm{rad}$ at $22.5 \mathrm{GHz}$. Using [12], the calculated RH-TL distributed series capacitance and inductance of the unit-cell hexagonal patch are $95 \mathrm{pF} / \mathrm{m}$ and $238 \mathrm{nH} / \mathrm{m}$, respectively. The shunt inductance $\mathrm{L}$ is $0.78 \mathrm{nH}$. For impedance matching between 50 input microstrip line and high input impedance of hexagon radiator, a simple coupling capacitor was utilized with gap $(S)$ of $0.44 \mathrm{~mm}$. The calculated coupling capacitance is about $49.8 \mathrm{fF}$. Input microstrip line serves as a reflector, endfire radiation characteristics are simply achieved and backward $\mathrm{TE}_{0}$ surface wave mode is suppressed. The lengths of each side of the top hexagon patch are properly chosen $3.8 \mathrm{~mm}\left(L_{1}\right), 2.4 \mathrm{~mm}\left(L_{2}\right)$ and $2.6 \mathrm{~mm}\left(L_{3}\right)$, respectively; the via diameter $(d)$ is $1 \mathrm{~mm}$, and the height and relative permittivity $(\varepsilon)$ of the substrate (RT/Duroid 5880) are $2.37 \mathrm{~mm}$ and 2.2, respectively.

\section{EXPERIMENTAL RESULTS}

In this paper, as a full-wave analysis method, ANSYS HFSS (FEM) is extensively used for simulations. High Frequency Structure Simulator (HFSS) is a complete solution 
for modelling arbitrarily-shaped, passive 3-D structures. It is a general purpose tool that can be used for a variety of electromagnetic (EM) modelling applications, including antenna design and analysis, machined-component design and analysis, circuit design and analysis and high-speed digital-circuit design and analysis. Moreover, cross check is also performed by using CST Microwave studio (FDTD). Figure 3 shows the $S_{11}$ parameters of the presented antennas with a hexagonal top patch. The resonance mode of the parasitic patch was scaled to operate at $21 \mathrm{GHz}, 26.4 \mathrm{GHz}$ and $29.8 \mathrm{GHz}$. The 10-dB bandwidth of the presented antenna is 14.7 $\mathrm{GHz}$ between $15.9 \mathrm{GHz}$ and $30.6 \mathrm{GHz}$, which corresponds to octave bandwidth. Figure 4 shows the maximum antenna gain and half-power beamwidth (HPBW). The maximum gain is $6 \mathrm{dBi}$ to $9.3 \mathrm{dBi}$ and the $3 \mathrm{~dB}$ HPBW varies from 42 degrees to 75 degrees. As can be seen, the radiation patterns are very similar and uniform over the whole operating frequencies.

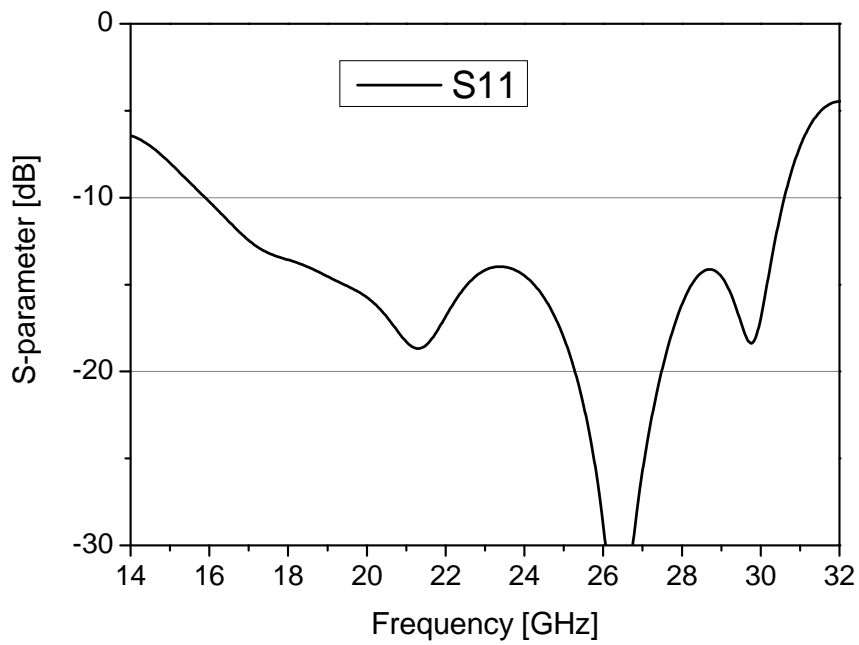

Fig. 3. Simulated S-parameter (S11).

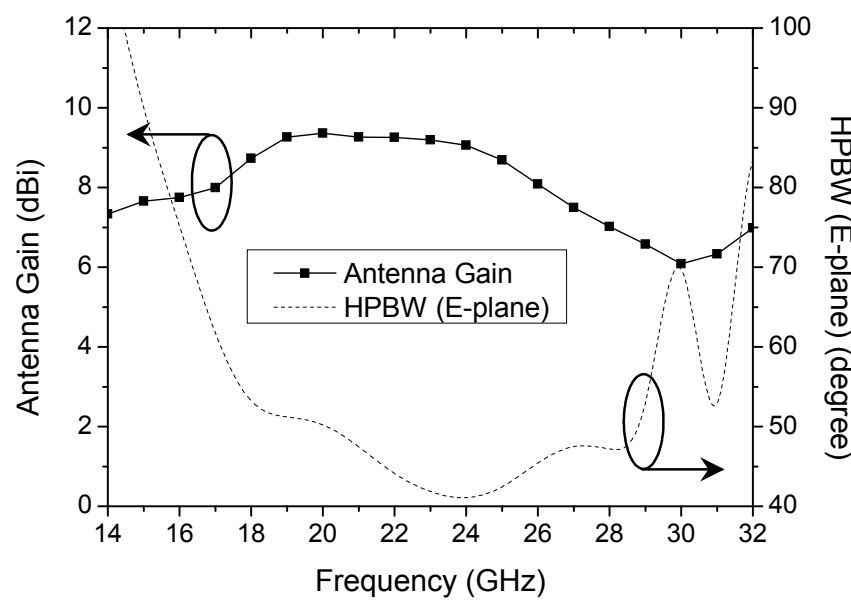

Fig. 4. Simulated maximum antenna gain and 3 dB HPBW of E-plane ( $x-y$ plane).

The 3-dimensional radiation patterns of the hexagonal-shaped top plates are plotted in Fig. 5 at $16 \mathrm{GHz}$, $20 \mathrm{GHz}, 24 \mathrm{GHz}$ and $28 \mathrm{GHz}$. From $16 \mathrm{GHz}$ to $28 \mathrm{GHz}$, we can see the broadside-to-endfire scanning capability of the proposed antenna. The E-field distributions at $16 \mathrm{GHz}, 20$ $\mathrm{GHz}, 24 \mathrm{GHz}$ and $28 \mathrm{GHz}$ on the surface of the antenna were investigated as shown in Fig. 6.

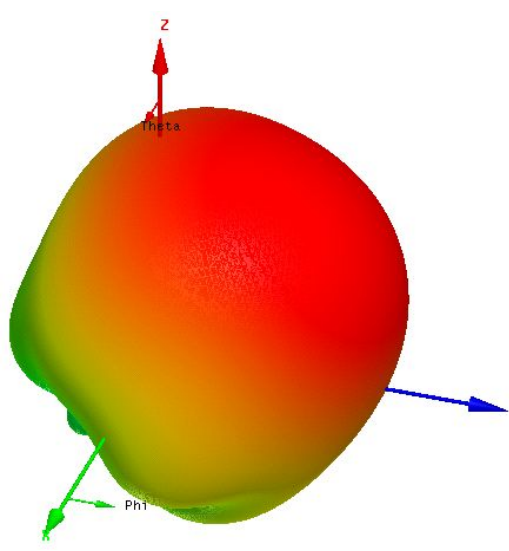

$\mathrm{dBi}($ Gain)

(a)

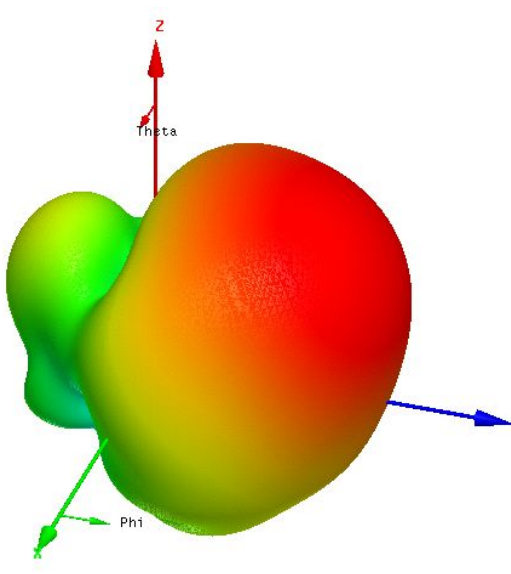

$\mathrm{dBi}($ Gain)

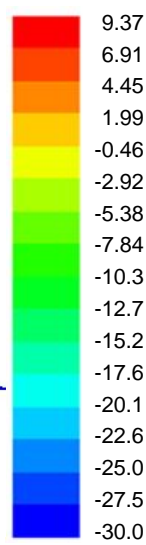

(b)

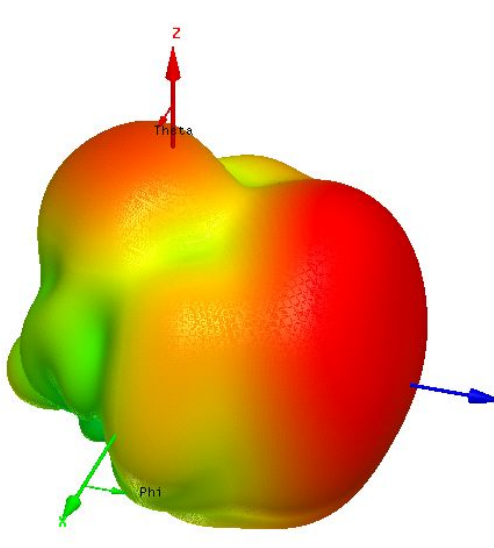

$\mathrm{dBi}($ Gain)

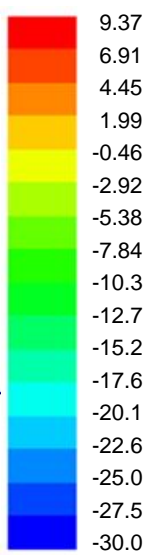

(c)

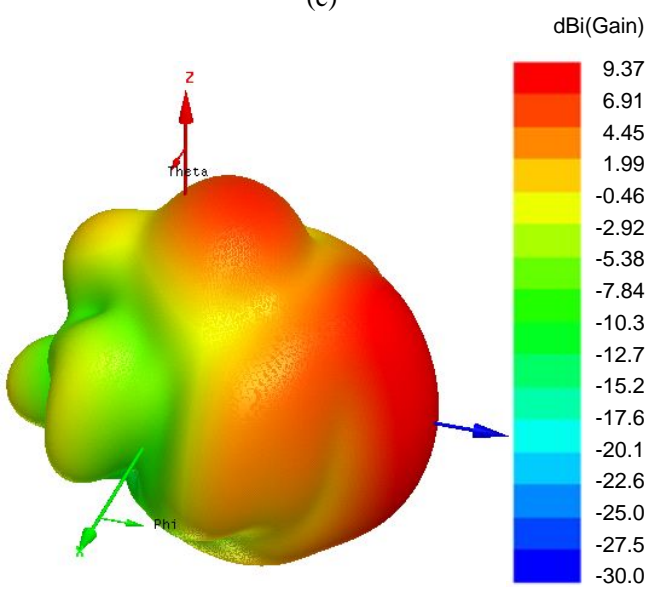

(d)

Fig. 5. Simulated 3D radiation patterns at (a) $16 \mathrm{GHz}$, (b) $20 \mathrm{GHz}$, (c) $24 \mathrm{GHz}$, (d) $28 \mathrm{GHz}$. 


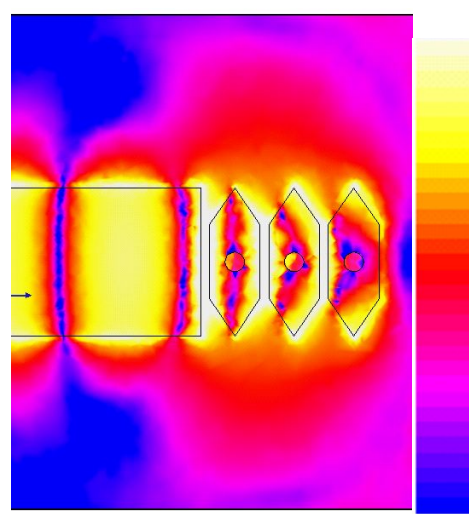

E-Field $(\mathrm{V} / \mathrm{m})$ $10.0 \mathrm{e} 10^{\wedge} 3$ $7.83 \mathrm{e} 10^{\wedge} 3$ $6.13 \mathrm{e} 10^{\wedge} 3$ $4.80 \mathrm{e} 10^{\wedge} 3$ $2.30 \mathrm{e} 10^{\wedge} 3$ $1.80 \mathrm{e} 10^{\wedge} 3$

$1.41 \mathrm{e}^{1} 0^{\wedge} 3$

1.101013

$8.67 \mathrm{e} 10^{\wedge} 2$

$6.79 \mathrm{e} 10^{\wedge} 2$

$5.31 \mathrm{e} 10^{\wedge} 2$

$4.16 \mathrm{e} 10^{\wedge} 2$

$3.26 \mathrm{e} 10^{\wedge} 2$

$2.55 \mathrm{e} 10^{\wedge} 2$ $2.00 \mathrm{e} 10^{\wedge} 2$

(a)

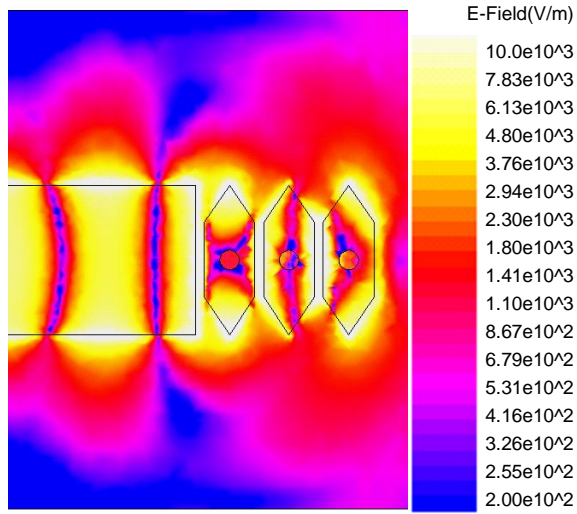

(b)

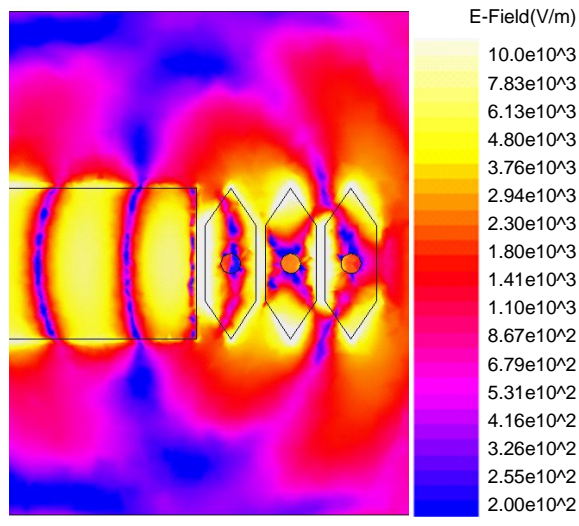

(c)

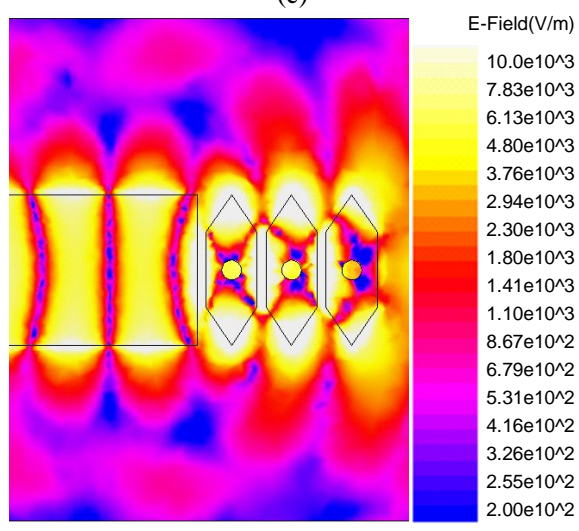

(d)

Fig. 6. E-field distributions at (a) $16 \mathrm{GHz}$, (b) $20 \mathrm{GHz}$, (c) $24 \mathrm{GHz}$, (d) $28 \mathrm{GHz}$ (x-y plane).
As it can be seen, at high-band frequencies, end-fired travelling wave are gradually generated and propagated to forward direction $(+y)$ of radiator. On the other hand, backward wave seem to be more suppressed by input feed line. The front-to-back $(\mathrm{F} / \mathrm{B})$ ratio is the ratio of the maximum forward directivity to that of the backward direction. Simulated F/B ratio ranges from $10 \mathrm{~dB}$ to $20 \mathrm{~dB}$.

\section{CONCLUSIONS}

In this paper, we present an octave band hexagonal-shaped patch antenna with mushroom-structured MTM. Owing to the effect of lengthening current paths on the hexagon patch and open CRLH TL structure, the proposed antenna demonstrates an octave (2:1) frequency bandwidth from $15.9 \mathrm{GHz}$ to $30.6 \mathrm{GHz}$ with the nominal radiation efficiency of $90 \%$. The simulated gain is from $6 \mathrm{dBi}$ to $9.3 \mathrm{dBi}$ and front-to-back ratio is more than $10 \mathrm{~dB}$. The overall antenna size with input feed line is $20 \mathrm{~mm} \times 21 \mathrm{~mm}$. The application areas are in broadband automotive radar and sensor applications.

\section{REFERENCES}

[1] M. J. Harry, Ground Penetrating Radar Theory and Applications. Oxford: U.K. Elsevier, 2009.

[2] D. J. Daniels, Ground Penetrating Radar. IEE, London: U.K, 2004 [Online]. Available: http://dx.doi.org/10.1049/PBRA015E

[3] B. Wu, Y. Ji, G. Fang, "Analysis of GPR UWB half-ellipse antennas with different heights of backed cavity above ground", IEEE Antennas and Wireless Propagation Letters, vol. 9, pp.130-133, 2010. [Online]. Available: http://dx.doi.org/10.1109/LAWP.2010.2044475

[4] V. G. Veselago, "The electrodynamics of substances with simultaneously negative values of $\varepsilon$ and $\mu$ ", Soviet Physics Uspekhi., vol. 10, no. 4, pp. 509-514, 1968. [Online]. Available: http://dx.doi.org/10.1070/PU1968v010n04ABEH003699

[5] C. Caloz, T. Itoh, "Application of the transmission line theory of left-handed $(\mathrm{LH})$ materials to the realization of a microstrip $\mathrm{LH}$ transmission line", IEEE-APS Int'l Symp. Digest, vol. 2, pp. 412-415 2002. [Online]. Available: http://dx.doi.org/10.1109/aps.2002. 1016111

[6] C. Caloz, H. Okabe, T. Iwai, T. Itoh, "Transmission line approach of left-handed (LH) materials", Proc. USNC/URSI National Radio Science Meeting, 2002, vol. 1, no. 39.

[7] S. K. Sharma, N. Jacob, L. Shafai, "Low profile wide band slot antenna for wireless communications", in Proc. IEEE AP-S Int. Symp., 2004 pp. 390-393.

[8] T. Huynh, K. F. Lee, "Single-layer single-patch wideband microstrip antenna", Electron. Lett., vol. 31, no. 16, pp. 1310-1312, 1995. [Online]. Available: http://dx.doi.org/10.1049/el:19950950

[9] R. Q. Lee, K. F. Lee, J. Bobinchak, "Characteristic of a two-layer electromagnetically coupled rectangular patch antenna", Electron. Lett., vol. 23, no. 20, pp. 1070-1071, 1987. [Online]. Available: http://dx.doi.org/10.1049/el:19870748

[10] L. Liu, C. Caloz, T. Itoh, "Dominant mode (DM) leaky wave antenna with backfire-to-end scanning capability", Electron. Lett., vol. 38 , no. 23, pp. 1414-1416, 2002. [Online]. Available: http://dx.doi.org/ 10.1049/el:20020977

[11] C. Caloz, T. Itoh, "Novel microwave devices and structures based on the transmission line approach of meta-materials", in Proc. IEEE-MTT Int. Symp., 2004, vol. 1, no. 9, pp. 195-198.

[12] M. S. Majedi, A. R. Attari1, "Dual-band resonance antennas using epsilon negative transmission line", IET Microw. Antennas Propag., vol. 7, no. 4, pp. 259-267, 2013. [Online]. Available: http://dx.doi.org/ 10.1049/iet-map.2012.0542 